(2) Open Access Full Text Article

\title{
Issues with Intravitreal Administration of Anti-VEGF Drugs
}

This article was published in the following Dove Press journal:

Clinical Ophthalmology

\author{
Marc Schargus ${ }^{1,2}$ \\ Andreas Frings 1,3 \\ 'Department of Ophthalmology, Heinrich \\ Heine University, Duesseldorf, Germany; \\ ${ }^{2}$ Department of Ophthalmology, \\ Asklepios Hospital Nord-Heidberg, \\ Hamburg, Germany; ${ }^{3}$ Augenarztpraxis \\ Dr. Frings, Nuremberg, Germany
}

Correspondence: Marc Schargus

Department of Ophthalmology, Heinrich-

Heine-University Düsseldorf,

Moorenstraße 5, 40225, Düsseldorf,

Germany

Tel +49-177-6272724

Email marc.schargus@gmx.de
Purpose: To provide an update of summary of risk factors and side effects of long-term use and contamination of intravitreal anti-VEGF injections.

Methods: We reviewed relevant publications of the topic of contamination of anti-VEGF injections and long-term side effects due to this reason.

Results: Due to the long-term use of anti-VEGF drugs and the higher number of injections worldwide, various studies have shown side effects in recent years, ranging from increased intraocular pressure to visual disturbing silicone oil vesicles in the vitreous cavity. Several studies have demonstrated that both the drug and the processing, storage, environmental factors and the material and design of the syringes have a decisive influence on these side effects.

Conclusion: The risks of deposits from syringes in the eye can be significantly reduced by various optimizations in transport, storage and syringe and cannula selection.

Keywords: contamination, intraocular pressure, protein particles, silicone oil, syringes, macular diseases

\section{Introduction}

Since 2005 the intravitreal anti-vascular endothelial growth factor (VEGF) drug bevacizumab (Avastin ${ }^{\circledR}$; Genentech, USA) and after approval the agents ranibizumab (Lucentis ${ }^{\circledR}$; Genentech, San Francisco, CA, USA) and aflibercept (Eylea ${ }^{\circledR}$; Regeneron Pharmaceuticals, Inc., Tarrytown, NY, USA) are used to treat several types of retinal vascular disorders. Currently, choroidal neovascularization (CNV) due to age-related macular degeneration, macular edema due to retinal vein occlusion, diabetic macular edema, CNV due to pathological myopia, secondary CNVs and retinopathy of prematurity are approved treatment targets. Injection intervals in active lesions are typically 4 weeks while several treatment schemes are in use to extend this range (eg, Treat and Extend [T\&E] injection scheme). ${ }^{1-3}$ New drugs such as brolucizumab (Beovu ${ }^{\circledR}$; Novartis, Basel, Switzerland) and Abicipar pegol (Abicipar $^{\circledR}$, Allergan, Dublin, Ireland) have already been approved or are about to be approved. Brolucizumab (Beovu $\left.{ }^{\circledR}\right)$ is a low molecular weight humanized singlechain variable (scFv) antibody fragment VEGF. This new agent binds and inhibits three main isoforms of VEGF-A (VEGF 110, VEGF 121 and VEGF 165). In addition, it prevents interaction with VEGFR-1 and VEGFR-2 receptor proteins, thereby suppressing vascular permeability, endothelial cell proliferation and neovascularization. Brolucizumab has a higher affinity than bevacizumab for human VEGF-A and a comparable affinity to ranibizumab. It should be administered every 
8-12 weeks. ${ }^{4}$ Abicipar-Pegol has a higher affinity and longer intraocular half-life than ranibizumab ( $>13$ days vs 7.2 days). This suggests that this new drug can also be used as a therapeutic agent with a longer duration and less frequent injections. 5,6

The number of patients in need of an anti-VEGF treatment is expected to dramatically increase in the coming years due to new indications and an aging population. ${ }^{7}$ Recently, data from the DRCR.net Protocol S study have led to the approval of ranibizumab for the treatment of proliferative diabetic retinopathy. ${ }^{8}$ As these patients are younger on average and require even longer treatment cycles than wet AMD patients, the number of injections per affected eye will continue increasing in the future. Cumulative risks from frequent injections are the result.

The three approved medicinal substances are currently prepared, stored and distributed in four different containers. Ranibizumab is supplied in two possible containers. Primarily, the single-dose vial has been introduced to the market, which is drawn into a syringe shortly before injection in the operating theatre. Since 2013, a pre-filled syringe has been available in the European Union, which was also approved by the US Food and Drug Administration (FDA) in 2016. In Central Europe, the prefilled syringe is currently the most commonly used syringe. ${ }^{9,10}$ Brolucizumab and aflibercept are currently supplied as originally ranibizumab as a single-dose vial, which is drawn into a syringe immediately before injection. A pre-filled syringe, as used for ranibizumab, will be used for brolucizumab in Europe. By August 2019 Regeneron announced a prefilled syringe that should be available by the end of the year 2019 in the US. ${ }^{11}$ Bevacizumab has not yet been approved as an intravitreal drug. The manufacturer is not expected to seek approval. Bevacizumab is therefore taken from larger vials, which are used and approved for systemic chemotherapy, by compounding pharmacies and then filled into several syringes. These syringes must be stored in the refrigerator at approx. $2^{\circ} \mathrm{C}$ to $8^{\circ} \mathrm{C}$ before use ${ }^{12}$ (Figure 1). Theoretically, any of these processing steps (compounding, refilling, inadvertent freeze and thaw) carries an increased risk for aggregate and particle generation.

Due to the high cost of intravitreal anti-VEGF drugs, compounding pharmacies around the world separate all these drugs in order to be able to use the entire quantity of active ingredients of the vials or syringes. The approved drugs are thus taken from their transport or injection containers with as little loss as possible in order to make them

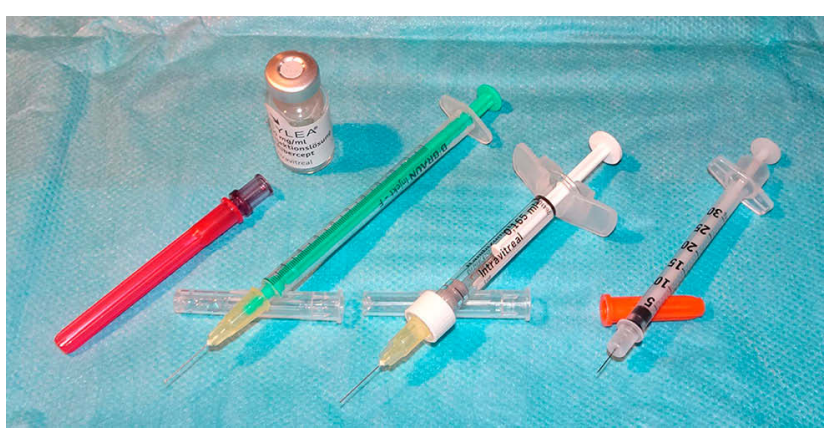

Figure I Examples of three different intravitreal injection syringes. Left: filter cannula and vial of aflibercept with Braun syringe with plugin 30G needle, middle: ranibizumab syringe with luer lock $30 \mathrm{G}$ needle design, right: low volume loss compound pharmacy 30G staked-on needle syringe.

available to surgeons in even lower-loss syringes. In thirdworld countries, bevacizumab is sometimes repacked directly by ophthalmic surgeons, which entails additional risks due to the lack of standardization and suboptimal conditions.

Currently, there are no worldwide standards in syringe preparation. Contaminations can easily occur and this depends on many factors. Even the volume of an antiVEGF injection can vary up to $25 \%$ due to the different syringe systems as shown by Loewenstein et al. ${ }^{13}$

This review summarizes previous studies on risk factors and side effects of anti-VEGF injections and their possible solutions.

\section{Possible Side Effects from Intravitreal Injections Elevated Intraocular Pressure}

After intravitreal injection, a short-term increase in IOP often occurs, which can be explained by the injection of the fluid volume into the vitreous body. ${ }^{14,15}$ The described long-term IOP increases have been clearly demonstrated in various large studies and case reports and case series, but the mechanism has not yet been finally clarified. For the first time, Bakri et al $^{16}$ reported a long-term IOP increase after ranibizumab injections, which required medical treatment in a small series of four patients, and Kahook et al reported similar findings in six patients. ${ }^{17}$ Over the years, several other studies have been published with varying numbers of patients reporting the prevalence of longterm IOP increase after treatment with bevacizumab, ranibizumab or aflibercept injections. ${ }^{17-30}$

One of the studies with the largest number of patients (retrospective analysis of VIEW 1 and 2 study data from a total of 2457 patients) showed a significantly higher IOP 
increase in eyes after ranibizumab treatment than in patients after aflibercept treatment. ${ }^{31}$

In 2016 Zhou et al conducted a meta-analysis of 5 randomized controlled trials (RCT) in 1428 patients and 8358 patients from 17 non-RCTs. ${ }^{32}$ The risk of a sustained increase in IOP after not excluding existing glaucoma patients was higher than the risk after excluding existing glaucoma patients. In non-RCTs, the pooled prevalence of elevated IOP was $4.7 \%$ regardless of the diagnostic criteria. Zhou et al found a pooled prevalence of elevated IOP in $8.3 \%$ with criteria of IOP above $22 \mathrm{mmHg}$ and 5 $\mathrm{mmHg}$ above baseline on 2 visits during follow-up. Additionally, they could show that the number of intravitreal anti-VEGF injections is associated with an increased risk of sustained IOP elevation (in this study more than 29 injections). In conclusion, Zhou calculated a 2-fold risk of developing elevated IOP after repeated intravitreal injections of anti-VEGF agents. ${ }^{32}$

Unfortunately, all published studies that evaluated IOP elevations after anti-VEGF injections have been retrospectively evaluated and are therefore of limited value.

In order to explain the clinically significant IOP increase after anti-VEGF treatment, several hypotheses were put forward. These include the theory of mechanical trauma of the trabecular meshwork due to recurrent IOP peaks caused by the injections and a decrease in aqueous humor outflow due to VEGF blockade, possibly caused by inhibition of nitric oxide synthesis or by inflammation or obstruction by silicone microdroplets or high molecular weight protein aggregates. ${ }^{33-35}$ A possible toxic effect of the anti-VEGF drugs on the trabecular meshwork was also postulated, but even at much higher doses than clinically used, there is no toxic effect in laboratory tests. ${ }^{36}$

The different molecular sizes (bevacizumab has a molecular weight of $149 \mathrm{kDa}$, aflibercept of $115 \mathrm{kDa}$ and ranibizumab of $48 \mathrm{kDa}$ ) were also assumed to be the cause of the intraocular pressure increases. In this theory, it is assumed that the substances accumulate in the trabecular meshwork mainly after long-term repeated administration, leading to a reduction of the outflow possibilities via direct or indirect obstruction. A direct obstruction could also be caused by the accumulation of silicone oil droplets or protein aggregates from the needle tips and syringes used. ${ }^{12,35,37}$ In a laboratory analysis of repackaged bevacizumab from three different compounding pharmacies in the USA, Kahook et al were able to detect protein aggregates as well as particles $\geq 1 \mu \mathrm{m} .{ }^{12}$
Wen et al performed two analyses to investigate if prolonged exposure to anti-VEGF therapy is a risk factor for a reduction in outflow facility and if patients with ocular hypertension were more likely to experience a substantial reduction in outflow facility from antiVEGF treatment. ${ }^{38}$ The study results from 40 examined patients showed a moderate reduction of outflow facility in eyes with a high number of injections $(\geq 20)$ compared to their non-injected fellow eyes. These findings suggest that increasing numbers of anti-VEGF injections are associated with a modest decline in the outflow facility on average. In patients with baseline ocular hypertension, the injected eye consistently demonstrated almost a 2 -fold reduction in outflow facility compared to the non-injected eye. From these data, Wen et al concluded that ocular hypertension is an additional risk factor for facility reduction in response to anti-VEGF therapy. The exact mechanism of outflow reduction remains unknown.

\section{Intraocular Inflammation and Endophthalmitis}

Since intravitreal administration takes place under sterile surgical conditions in most countries, the risk of surgical complications has been greatly reduced, with bacterial endophthalmitis being by far the most devastating.

Several small series of intraocular inflammation after intravitreal injections of anti-VEGF presenting as uveitis have been described in the literature. ${ }^{39}$ Non-infectious vitritis has been reported by Williams et al in $0.10 \%$ after 66,356 bevacizumab injections, $0.02 \%$ after 26,161 ranibizumab injections and $0.16 \%$ after 8071 aflibercept injections. ${ }^{40} \mathrm{~A}$ true immune reaction to the anti-VEGF drug itself might be the explanation for the sporadic cases in which a rechallenge does result in a recurrent uveitis event. The clustered cases described in the literature however probably resulted from a problem in the preparation or delivery process.

Two large population-based studies have been published in recent years analyzing the endophthalmitis rate after intravitreal injection. ${ }^{41,42}$

Baudin et al evaluated the incidence of acute endophthalmitis after intravitreal injection of corticosteroids or anti-VEGF agents in a nationwide study in France. Taking only the anti-VEGF injections into account, 1.67 million injections were evaluated from the French medical-administrative database. The total incidence of endophthalmitis was $0.020 \%$, whereas the endophthalmitis 
incidence of prefilled ranibizumab syringes was only $0.013 \%$. The prefilled ranibizumab syringe therefore had decreased the rate of endophthalmitis by $40 \%$ compared with ranibizumab in a vial and by $46 \%$ for aflibercept in a vial. ${ }^{41}$

Storey et al analysed 243754 injections of ranibizumab in 10 centers in the USA (2016 to 2017) and Japan (2009 to 2017). ${ }^{42}$ The suspected rate of endophthalmitis with an injection of ranibizumab in a vial was $0.026 \%$, the rate of culture-positive endophthalmitis was $0.013 \%$. The rate of suspicious endophthalmitis of prefilled syringes containing ranibizumab was $0.015 \%$ and $0.0026 \%$ for culture-positive endophthalmitis.

All population-based studies have numerous limitations that limit the interpretation of the results, not least due to the study design and the method of data collection. Nevertheless, these are the most comprehensive data ever published on this topic. Both studies suggest that the endophthalmitis rate can be significantly reduced by prefilled syringes. ${ }^{41,42}$

The main reason for decreased risk of endophthalmitis while using prefilled syringes is due to lack of need for transfer of drug into another syringe, thus reducing the manipulations. This results in improved safety. This reason is consistent with published endophthalmitis series associated with repackaged bevacizumab. ${ }^{43,44}$

Bande et al conducted a meta-analysis to investigate whether the prophylactic administration of antibiotics significantly reduces the endophthalmitis rate. This could not be confirmed by the analysis; on the contrary, the group with antibiotic administration showed a higher rate of endophthalmitis (1.7 times). Also, the location of the operation (operating theatre or outpatient clinic) did not play a role in the endophthalmitis rate. According to the current study situation, the peri- or postoperative administration of antibiotics can therefore be abandoned. Both findings have implications for patient comfort as well as for the cost and efficiency of intravitreal drug administration. ${ }^{45}$

\section{Silicone Oil Droplets and Protein Aggregates}

In addition to the active ingredient, other undesirable particles are injected into the eye. These include silicone oil droplets that detach themselves from the inner coating of the syringe walls (Figure 2). The manufacturing process of most commercially available syringes relies on siliconisation of the inner surface of the syringe barrel, which

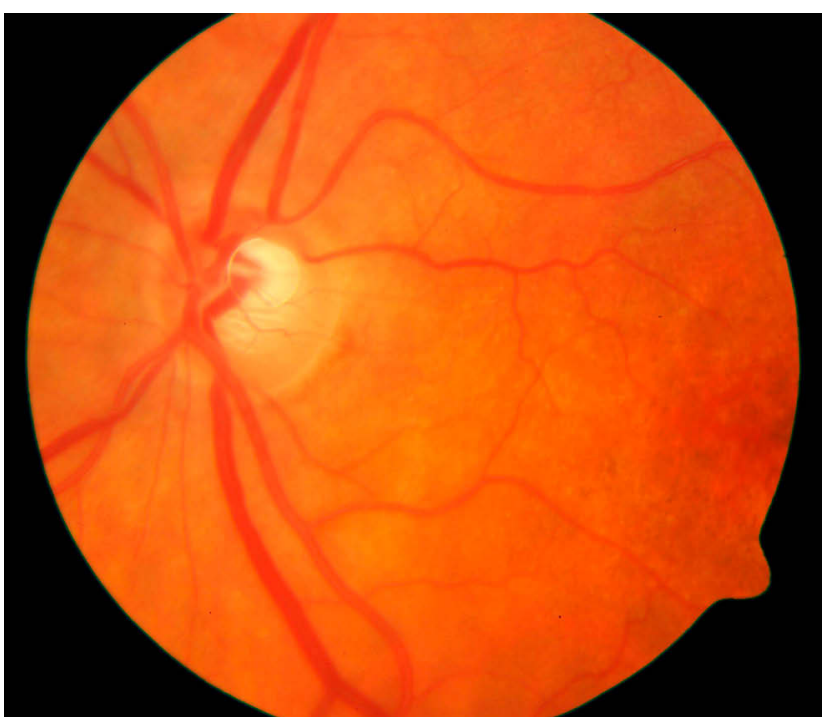

Figure 2 Prepapillary silicone oil droplet after 30 intravitreal injections of antiVEGF agent in a patient with wet AMD.

facilitates reduced force to initiate the movement of the plunger and its subsequent gliding. ${ }^{46}$ Furthermore, transport and storage can lead to aggregation of protein particles in the syringes.

In the American Academy of Ophthalmology's annual survey presented at the 2018 Chicago Annual Meeting, 1019 ophthalmologists (US American and international) were asked if they had seen silicone oil droplets after intravitreal injections of bevacizumab within the last year. A $60.4 \%$ of the US American and $27 \%$ of the international participants answered yes to this question, but without the silicone oil droplets being symptomatic for the patients. A $5.2 \%$ of the US American and $1.4 \%$ of the international ophthalmologists, however, have already had to perform vitrectomies due to symptomatic silicone oil vesicles and $1.8 \%$ (US) and $0.7 \%$ (international) of the ophthalmologists interviewed answered that some patients have sought legal action.

It has been known for some time that if silicone oil remains in the eye for more than 12 to 18 months, it can be trapped by the trabecular meshwork and the Müller cells of the retina; this is also believed to cause an increase in intraocular pressure. ${ }^{47}$

As already mentioned, Kahook et al already reported in a laboratory analysis of particles $\geq 1 \mu \mathrm{m}$ and protein aggregates in repackaged bevacizumab from three different compounding pharmacies in the USA. ${ }^{12}$ Khurana et al have estimated the incidence of presumed intraocular silicone oil droplets after intravitreal bevacizumab injection 
in their study population in the USA with insulin syringes to range from $0.03 \%$ (3230 injections) to $1.7 \%$ (3402 injections) at different time periods. ${ }^{48}$ Bakri et al were able to detect silicone oil droplets in 15 of 1529 injections in their patient population in the USA $(0.1 \%) .{ }^{35}$

On the other hand, Melo et al were able to detect a very high proportion of $68-76 \%$ with silicone oil droplets in the vitreous body after intravitreal injections in Brazil. The greater the number of injections, the higher was the likelihood of finding silicone oil. Attention is already drawn here to the clear differences in the syringe systems used in Brazil. ${ }^{49}$

Several studies have shown that flicking or agitation of the syringes results in a high amount of silicone oil droplets inside the syringes. ${ }^{37,50}$

The authors carried out further investigations on the particle count in two bevacizumab syringes filled by different compounding pharmacies (University and External), as well as the aflibercept vial and the ranibizumab ready-to -use syringe available in Germany. The transport route was also taken into account. ${ }^{51}$

The results showed that the quality of the repackaged bevacizumab from the university pharmacy, the aflibercept vial and the siliconized ranibizumab glass syringe were very similar. A much higher particle load (as seen in examination by light obscuration) was found in the bevacizumab repackaged by the external compounding pharmacy. One reason for the higher particle count could be the staked-in needle, which was only used for the repackaged drugs.

The contents of the ranibizumab pre-filled syringe showed the lowest number of protein-based submicronparticles in most analytical methods, although it should be noted that the concentration of proteins in the ranibizumab solution is expected to be lowest.

Ranibizumab shows the highest quality in terms of protein-based submicron particles in the majority of the analytical methods used. However, one should keep in mind that protein concentration in ranibizumab is the lowest one. In future studies comparing the ranibizumab prefilled-syringe with aflibercept vials, at least the correlation between particle numbers assumed in VIEW 1 and VIEW 2 study analyses can no longer be taken as a basis. The results showed that repackaging does not automatically lead to a worse quality in terms of particle burden compared to "original" products. Nevertheless, it was found that significant differences were already observed in two bevacizumab products repackaged from different compound pharmacies.

\section{Options to Reduce Contamination and Side Effects}

Hardly any syringe system is approved for intravitreal injections except the prefilled syringe of ranibizumab and aflibercept. For the time being, there will be no approved syringe system for the administration of bevacizumab without corresponding approval of the drug.

Moisseiev et al were able to demonstrate that different types of syringes, despite having the same labelling, deliver different volumes and thus deviate significantly from the values that the surgeon would like to administer during the injection. ${ }^{52}$ The three syringe models tested in this study injected a significantly higher volume than the intended $50 \mu \mathrm{L}$. In the study described, the syringe design with low dead space showed the highest reproducibility of the delivered volume. Sampat et al also found significant deviations between $3.8 \%$ and $6 \%$ from the planned injection volume of $0.05 \mathrm{~mL}$ when examining seven different syringe systems. ${ }^{53}$ This shows that it is almost impossible to inject the exact same amount of anti-VEGF into the eye. Since different syringes have different long stroke lengths, the shortest possible stroke length should be selected to minimize silicone abrasion on the inner walls of the syringe.

Compared to the staked-on syringe design, syringes with luer cone design have a significantly lower frequency of occurrence of intravitreal silicone oil droplets as demonstrated in the study "Standard Care versus COrticosteroid in REtinal Vein Occlusion" (SCORE). The authors attribute this to the increased residual space in the needle hub with the luer cone design. ${ }^{47}$

Luer Lock syringes preserve a residual volume of about $50 \mu \mathrm{L}$ inside the syringe. This prevents the residual volume, which probably contains the largest amount of silicone oil droplets from the walls of the coated syringe, from being injected into the eye. In contrast, the staked-on syringe design has no such residual space, so "squeegeed" silicone oil is injected into the eye if the plunger is pushed all the way down the barrel of the syringe. ${ }^{47}$

Therefore, syringes without staked-on needles should be used for maximum reduction of silicone oil particles even if these syringes are not the most reproducible in kind of the exact volume.

In the laboratory study by Liu et al both repackaged bevacizumab syringes from compounding pharmacies and direct samples of bevacizumab and ranibizumab vials were tested for the content of sub-visible particles and protein 
aggregates. ${ }^{37}$ Several interesting mechanical, temperature depended and temporal effects on individual bevacizumab syringes were investigated. Simulating shocks by 20 times throwing a cool box with syringes over $1.2 \mathrm{~m}$ to the ground, the number of particles in microflow imaging increased 4.5 times. (silicone oil particles and protein aggregates were not differentiated). Repeated freezing, as can occur with a narrow positioning on a cold pack, also led to a strong increase in particle count; at 5 times freezing/thawing cycle the particle count was increased 7.5 times. Exposure to light for 8 weeks increased the particle count by 2.5 times. Storage over 12 weeks under optimal conditions showed no significant change in particle number.

Light, mechanical shocks as well as repeated freezing and thawing increases the proportion of particles in bevacizumab syringes, sometimes considerably. Syringes should, therefore, be transported protected from light. Long transport routes inevitably increase the risk of repeated mechanical shocks and should, therefore, be avoided, especially if the transport service is not specialized in transporting sensitive medical goods. When packing transport boxes, scrupulous care should be taken to ensure that the syringes cannot come into direct contact with frozen cold packs and should be packed as shock proofed as possible.

Khurana et al described an increase in the incidence of intravitreal silicone oil droplets over a 7-month period observed in their practice in $2016 .{ }^{48}$ Other US physicians have reported similar findings. The compounding pharmacy denied any change in the preparation or handling of bevacizumab during the time in question, a change in the used insulin syringes may explain increased silicone oil droplets but there was no information given from the manufacturer.

Surgeons cannot monitor and control the entire production process. Therefore, it is important to have a trustworthy pharmacy at hand, which informs the surgeon in case of changes in the production process, so that the surgeon can immediately detect possible abnormalities.

The ready-to-use syringe for ranibizumab introduced by Novartis in 2013 already has a number of advantages that set it apart from simple insulin syringes. These include a non-reactive borosilicate glass for optimized storage stability, a non-retractable plunger and a so-called "backed siliconization process" glass which reduces the amount of silicone on the inside of the syringe and thus also the transfer of silicone oil droplets into the injection solution. This allows a storage period of up to 3 years.

Compared to vials with separately supplied syringes and filter cannulas, pre-filled syringes have many advantages. These include accuracy, affordability, improved convenience, safety and sterility. But the benefits are even more far-reaching: the use of pre-filled syringes reduces the risk of needle stick injuries that can occur when taking medication from vials, improves dosing accuracy and reduces dosing errors. Economically speaking, the reduced overfilling of the syringe also makes sense because the minimization of drug waste means that costs can be reduced. Further advantages of the ready-to-use syringe are the significant time savings in the preparation of the syringes but also the faster elimination of air bubbles from the substances to be injected. The ready-to-use syringe simplifies the injection considerably, so that in some countries, injections by non-ophthalmologists can also be carried out more easily, quickly and safely. ${ }^{54,55}$

However, glass syringes such as the approved ranibizumab pre-filled syringe are prone to breakage and have a relatively large weight compared to plastic syringes.

Lode et al recently published a method for refilling anti-VEGF drugs into silicone oil-free plastic syringes in Norway. ${ }^{56}$ In the test performed, a stability of the antiVEGF drugs over 1 week could be proven. Further studies are necessary to make this desirable filling method available to other countries.

\section{Conclusion}

The described studies show how important it is that ophthalmologists who treat patients with intravitreal injections must be aware of the possible changes and described findings. Monitoring of patients with known elevated intraocular pressure, such as ocular hypertension or patients with known glaucoma, is strongly recommended. Larger, prospective studies are needed to better understand the exact consequences of long-term therapy with antiVEGF drugs. Furthermore, the occurrence of silicone oil droplets in the vitreous body after multiple anti-VEGF injections should be closely observed. Patients should also be informed, in addition to the known other risks, that an accumulation of silicone oil droplets can cause visual impairment. The handling of anti-VEGF syringes contributes significantly to the number of injected particles and should, therefore, be handled with care. The choice of syringes used should be discussed and modified with the compounding pharmacy in accordance with the properties 
outlined in this article. In future, pre-filled silicone-free plastic syringes with exact volume dispensing for optimum patient treatment would be desirable.

\section{Disclosure}

The authors report no conflicts of interest in this work.

\section{References}

1. Berg K, Hadzalic E, Gjertsen I, et al. Ranibizumab or bevacizumab for neovascular age-related macular degeneration according to the lucentis compared to avastin study treat-and-extend protocol: two-year results. Ophthalmology. 2016;123(1):51-59. doi:10.1016/j. ophtha.2015.09.018

2. Brown DM, Kaiser PK, Michels M, et al. Ranibizumab versus verteporfin for neovascular age-related macular degeneration. $N$ Engl $J$ Med. 2006;355(14):1432-1444. doi:10.1056/NEJMoa062655

3. Heier JS, Brown DM, Chong V, et al. Intravitreal aflibercept (VEGF trap-eye) in wet age-related macular degeneration. Ophthalmology. 2012;119(12):2537-2548. doi:10.1016/j.ophtha.2012.09.006

4. Tietz J, Spohn G, Schmid G, et al. Affinity and potency of RTH258 (ESBA1008), a novel inhibitor of vascular endothelial growth factor a for the treatment of retinal disorders [abstract]. Invest Ophthalmol Vis Sci.2015;56(7):1501.

5. Krohne TU, Liu Z, Holz FG, Meyer CH. Intraocular pharmacokinetics of ranibizumab following a single intravitreal injection in humans. Am J Ophthalmol. 2012;154(4):682-686. (). doi:10.1016/j. ajo.2012.03.047

6. Rodrigues GA, Mason M, Christie L-A, et al. Functional characterization of abicipar-pegol, an Anti-VEGF DARPin therapeutic that potently inhibits angiogenesis and vascular permeability. Investigative Opthalmology \& Visual Science. 2018;59(15):5836-58 46. doi:10.1167/iovs.18-25307

7. Wong WL, Su X, Li X, et al. Global prevalence of age-related macular degeneration and disease burden projection for 2020 and 2040: a systematic review and meta-analysis. Lancet Glob Health 2014;2(2):e106-116. doi:10.1016/S2214-109X(13)70145-1

8. Sun JK, Glassman AR, Beaulieu WT, et al. Rationale and application of the protocol S anti-vascular endothelial growth factor algorithm for proliferative diabetic retinopathy. Ophthalmology. 2019;126 (1):87-95. doi:10.1016/j.ophtha.2018.08.001

9. FDA approves Lucentis (ranibizumab injection) prefilled syringe. 2016. Available from: http://www.roche.com/de/investors/updates/ inv-update-2016-10-17.htm. Accessed March 10, 2020.

10. Michaud J-E, Sigg J, Boado L, et al. Ranibizumab pre-filled syringe approved in the European Union: innovation to improve dose accuracy, reduce potential infection risk, and offer more efficient treatment administration. Invest Ophthalmol Vis Sci. 2014;55(13):1949.

11. EYLEA $^{\circledR}$ (aflibercept) Injection full U.S. Prescribing Information. Regeneron Pharmaceuticals, Inc. August 2019. Available from: https://www.regeneron.com/sites/default/files/EYLEA_FPI.pdf. Accessed December 28, 2019

12. Kahook MY, Liu L, Ruzycki P, et al. High-molecular-weight aggregates in repackaged bevacizumab. Retina. 2010;30(6):887-892. doi:10.1097/IAE.0b013e3181d50cea

13. Loewenstein I, Goldstein M, Moisseiev J, Moisseiev E. Accuracy and precision of intravitreal injections of anti-vascular endothelial growth factor agents in real life: what is actually in the syringe? Retina. 2019;39(7):1385-1391. doi:10.1097/IAE.0000000000002170

14. Kiddee W, Montriwet M. Intraocular pressure changes in non-glaucomatous patients receiving intravitreal anti-vascular endothelial growth factor agents. PLoS One. 2015;10(9):e0137833. doi:10.1371/journal.pone. 0137833
15. Farhood QK, Twfeeq SM. Short-term intraocular pressure changes after intravitreal injection of bevacizumab in diabetic retinopathy patients. Clin Ophthalmol. 2014;8:599-604. doi:10.2147/OPTH

16. Bakri SJ, McCannel CA, Edwards AO, Moshfeghi DM. Persistent ocular hypertension following intravitreal ranibizumab. Graefe's Archive Clin Exp Ophthalmol. 2008;246(7):955-958. doi:10.1007/ s00417-008-0819-2

17. Kahook MY, Kimura AE, Wong LJ, Ammar DA, Maycotte MA, Mandava N. Sustained elevation in intraocular pressure associated with intravitreal bevacizumab injections. Ophthalmic Surg Lasers Imaging. 2009;40(3):293-295. doi:10.3928/154288 77-20090430-12

18. Abedi G, Adelman RA, Salim S. Incidence and management of elevated intraocular pressure with antivascular endothelial growth factor agents. Semin Ophthalmol. 2013;28(3):126-130. doi:10.3109/ 08820538.2013 .771195

19. Adelman RA, Zheng Q, Mayer HR. Persistent ocular hypertension following intravitreal bevacizumab and ranibizumab injections. J Ocul Pharmacol Ther. 2010;26(1):105-110. doi:10.1089/jop.20 09.0076

20. Good TJ, Kimura AE, Mandava N, Kahook MY. Sustained elevation of intraocular pressure after intravitreal injections of anti-VEGF agents. Br J Ophthalmol. 2011;95(8):1111-1114. doi:10.1136/bjo.20 10.180729

21. Hoang QV, Mendonca LS, Della Torre KE, Jung JJ, Tsuang AJ, Freund KB. Effect on intraocular pressure in patients receiving unilateral intravitreal anti-vascular endothelial growth factor injections. Ophthalmology. 2012;119(2):321-326. doi:10.1016/j.ophtha.2011.08.011

22. Hoang QV, Tsuang AJ, Gelman R, et al. Clinical predictors of sustained intraocular pressure elevation due to intravitreal anti-vascular endothelial growth factor therapy. Retina. 2013;33 (1):179-187. doi:10.1097/IAE.0b013e318261a6f7

23. Wehrli SJ, Tawse K, Levin MH, Zaidi A, Pistilli M, Brucker AJ. A lack of delayed intraocular pressure elevation in patients treated with intravitreal injection of bevacizumab and ranibizumab. Retina. 2012;32(7):1295-1301. doi:10.1097/IAE.0b013e31823f0c95

24. Sniegowski M, Mandava N, Kahook MY. Sustained intraocular pressure elevation after intravitreal injection of bevacizumab and ranibizumab associated with trabeculitis. Open Ophthalmol J. 2010;4: 28-29. doi:10.2174/1874364101004010028

25. Matsubara H, Miyata R, Kobayashi M, Tsukitome H, Ikesugi K, Kondo M. A case of sustained intraocular pressure elevation after multiple intravitreal injection of ranibizumab and aflibercept for neovascular age-related macular degeneration. Case Rep Ophthalmol. 2016;7(1):230-236. doi:10.1159/000446016

26. Mathalone N, Arodi-golan A, Sar S, et al. Sustained elevation of intraocular pressure after intravitreal injections of bevacizumab in eyes with neovascular age-related macular degeneration. Graefe's Archive Clin Exp Ophthalmol. 2012;250(10):1435-1440. doi:10.10 07/s00417-012-1981-0

27. Dedania VS, Bakri SJ. Sustained elevation of intraocular pressure after intravitreal anti-vegf agents: what is the evidence? Retina. 2015;35(5):841-858.

28. Bressler SB, Almukhtar T, Bhorade A, et al. Repeated intravitreous ranibizumab injections for diabetic macular edema and the risk of sustained elevation of intraocular pressure or the need for ocular hypotensive treatment. JAMA Ophthalmol. 2015;133(5):589-597. doi:10.1001/jamaophthalmol.2015.186

29. Baek SU, Park IW, Suh W. Long-term intraocular pressure changes after intravitreal injection of bevacizumab. Cutan Ocul Toxicol. 2016;35(4):310-314. doi:10.3109/15569527.2015.1124886

30. Rusu IM, Deobhakta A, Yoon D, et al. Intraocular pressure in patients with neovascular age-related macular degeneration switched to aflibercept injection after previous anti-vascular endothelial growth factor treatments. Retina. 2014;34(11):2161-2166. doi:10.1097/ IAE.0000000000000264 
31. Freund KB, Hoang QV, Saroj N, Thompson D. Intraocular pressure in patients with neovascular age-related macular degeneration receiving intravitreal aflibercept or ranibizumab. Ophthalmology. 2015;122 (9):1802-1810. doi:10.1016/j.ophtha.2015.04.018

32. Zhou Y, Zhou M, Xia S, Jing Q, Gao L. Sustained elevation of intraocular pressure associated with intravitreal administration of anti-vascular endothelial growth factor: a systematic review and meta-analysis. Sci Rep. 2016;6(1):39301. doi:10.1038/srep39301

33. Bakri SJ, Moshfeghi DM, Francom S, et al. Intraocular pressure in eyes receiving monthly ranibizumab in 2 pivotal age-related macular degeneration clinical trials. Ophthalmology. 2014;121(5):1102-1108. doi:10.1016/j.ophtha.2013.11.029

34. Ricca AM, Morshedi RG, Wirostko BM. High intraocular pressure following anti-vascular endothelial growth factor therapy: proposed pathophysiology due to altered nitric oxide metabolism. J Ocul Pharmacol Ther. 2015;31(1):2-10. doi:10.1089/jop.2014.0062

35. Bakri SJ, Ekdawi NS. Intravitreal silicone oil droplets after intravitreal drug injections. Retina. 2008;28(7):996-1001. doi:10.1097/ IAE.0b013e31816c6868

36. Kahook MY, Ammar DA. In vitro effects of antivascular endothelial growth factors on cultured human trabecular meshwork cells. J Glaucoma. 2010;19(7):437-441. doi:10.1097/IJG.0b013e3181ca74de

37. Liu L, Ammar DA, Ross LA, Mandava N, Kahook MY, Carpenter JF. Silicone oil microdroplets and protein aggregates in repackaged bevacizumab and ranibizumab: effects of long-term storage and product mishandling. Invest Ophthalmol Vis Sci. 2011;52(2):1023-1034. doi:10.1167/iovs.10-6431

38. Wen JC, Reina-torres E, Sherwood JM, et al. Intravitreal anti-VEGF injections reduce aqueous outflow facility in patients with neovascular age-related macular degeneration. Invest Ophthalmol Vis Sci. 2017;58(3):1893-1898. doi:10.1167/iovs.16-20786

39. Trivizki O, Schwartz S, Negri N, Loewenstein A, Rabina G, Shulman S. Noninfectious inflammatory response following intravitreal bevacizumab injections: description of a cluster of cases in two centers and a review of the literature. Ophthalmologica. 2018;240 (3):163-166. doi:10.1159/000492681

40. Williams PD, Chong D, Fuller T, Callanan D. Noninfectious vitritis after intravitreal injection of anti-vegf agents: variations in rates and presentation by medication. Retina. 2016;36(5):909-913. doi:10.10 97/IAE.0000000000000801

41. Baudin F, Benzenine E, Mariet AS, et al. Association of acute endophthalmitis with intravitreal injections of corticosteroids or anti-vascular growth factor agents in a nationwide study in France. JAMA Ophthalmol. 2018;136(12):1352-1358. doi:10.1001/ jamaophthalmol.2018.3939

42. Storey PP, Tauqeer Z, Yonekawa Y, et al. The impact of prefilled syringes on endophthalmitis following intravitreal injection of ranibizumab. Am J Ophthalmol. 2019;199:200-208. doi:10.1016/j. ajo.2018.11.023

43. Edison LS, Dishman HO, Tobin-d'angelo MJ, Allen CR, Guh AY, Drenzek CL. Endophthalmitis outbreak associated with repackaged bevacizumab. Emerg Infect Dis. 2015;21(1):171-173. doi:10.3201/ eid2101.141040
44. Sheyman AT, Cohen BZ, Friedman AH, Ackert JM. An outbreak of fungal endophthalmitis after intravitreal injection of compounded combined bevacizumab and triamcinolone. JAMA Ophthalmol. 2013;131(7):864-869. doi:10.1001/jamaophthalmol.2013.88

45. Bande MF, Mansilla R, Pata MP, et al. Intravitreal injections of anti-VEGF agents and antibiotic prophylaxis for endophthalmitis: a systematic review and meta-analysis. Sci Rep. 2017;7(1):18088. doi:10.1038/s41598-017-18412-9

46. Sacha GA, Saffell-clemmer W, Abram K, Akers MJ. Practical fundamentals of glass, rubber, and plastic sterile packaging systems. Pharm Dev Technol. 2010;15(1):6-34. doi:10.3109/108374509035 11178

47. Scott IU, Oden NL, VanVeldhuisen PC, et al. SCORE study report 7: incidence of intravitreal silicone oil droplets associated with staked-on vs luer cone syringe design. Am J Ophthalmol. 2009;148 (5):725-732 e727. doi:10.1016/j.ajo.2009.06.004

48. Khurana RN, Chang LK, Porco TC. Incidence of presumed silicone oil droplets in the vitreous cavity after intravitreal bevacizumab injection with insulin syringes. JAMA Ophthalmol. 2017;135(7):80 0-803. doi:10.1001/jamaophthalmol.2017.1815

49. Melo GB, Dias Junior C, Morais FB, et al. Prevalence of silicone oil droplets in eyes treated with intravitreal injection. Int J Retina Vitreous. 2019;5:34. doi:10.1186/s40942-019-0184-9

50. Melo GB, Dias Junior C, Carvalho MR, et al. Release of silicone oil droplets from syringes. Int J Retina Vitreous. 2019;5:1. doi:10.1186/ s40942-018-0153-8

51. Schargus M, Werner BP, Geerling G, Winter G. Contamination of anti-vegf drugs for intravitreal injection: how do repackaging and newly developed syringes affect the amount of silicone oil droplets and protein aggregates?. Retina. 2018;38(10):2088-2095.

52. Moisseiev E, Rudell J, Tieu EV, Yiu G. Effect of syringe design on the accuracy and precision of intravitreal injections of Anti-VEGF agents. Curr Eye Res. 2017;42(7):1059-1063. doi:10.1080/02713683. 2016.1276195

53. Sampat KM, Wolfe JD, Shah MK, Garg SJ. Accuracy and reproducibility of seven brands of small-volume syringes used for intraocular drug delivery. Ophthalmic Surg Lasers Imaging Retina. 2013;44 (4):385-389. doi:10.3928/23258160-20130601-02

54. Souied E, Nghiem-buffet S, Leteneux C, et al. Ranibizumab prefilled syringes: benefits of reduced syringe preparation times and less complex preparation procedures. Eur J Ophthalmol. 2015;25(6):52 9-534. doi:10.5301/ejo.5000629

55. Subhi Y, Kjer B, Munch IC. Prefilled syringes for intravitreal injection reduce preparation time. Dan Med J. 2016;63(4):A5214.

56. Lode HE, Gjølberg TT, Foss S, et al. A new method for pharmaceutical compounding and storage of anti-VEGF biologics for intravitreal use in silicone oil-free prefilled plastic syringes. Sci Rep. 2019;9 (1):18021. doi:10.1038/s41598-019-54226-7
Clinical Ophthalmology

\section{Publish your work in this journal}

Clinical Ophthalmology is an international, peer-reviewed journal covering all subspecialties within ophthalmology. Key topics include: Optometry; Visual science; Pharmacology and drug therapy in eye diseases; Basic Sciences; Primary and Secondary eye care; Patient Safety and Quality of Care Improvements. This journal is indexed on PubMed
Central and CAS, and is the official journal of The Society of Clinical Ophthalmology (SCO). The manuscript management system is completely online and includes a very quick and fair peer-review system, which is all easy to use. Visit http://www.dovepress.com/ testimonials.php to read real quotes from published authors. 